\title{
Reviewers in this issue
}

\section{Dr. M. A. Salam Khan}

Professor of Anaesthesiology, SICU

Bangladesh Institute of Research \& Rehabilitation in Diabetes,

Endocrine and Metabolic Disorder (BIRDEM) Academy \& Hospital, Dhaka, Bangladesh

\section{Dr. S. M. Fazlul Karim}

Professor of Biochemistry

Delta Medical College, Dhaka, Bangladesh

Dr. Md. Rafiqul Islam

Professor of Paediatrics

Delta Medical College, Dhaka, Bangladesh

\section{Dr. Shahida Choudhury}

Professor of Psychiatry

Delta Medical College, Dhaka, Bangladesh

\section{Dr. Md. Aminul Haque Khan}

Professor of Biochemistry

Enam Medical College, Savar, Dhaka, Bangladesh

\section{Dr. Shameem Ahmed}

Professor of Anatomy with Histology

Kumudini Womens Medical College, Tangail, Bangladesh

\section{Dr. Rehnuma Jahan}

Associate Consultant of Gynaecology \& Obstetrics

Square Hospital, Dhaka, Bangladesh

\section{Dr. Rezaul Farid Khan}

Associate Professor of Community Medicine

Delta Medical College, Dhaka, Bangladesh 